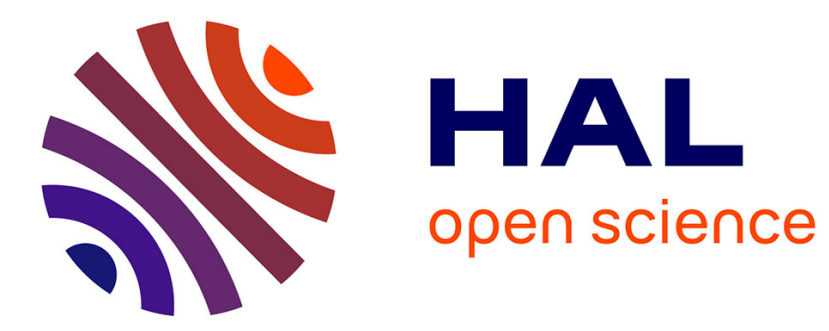

\title{
Les éleveurs face à la territorialisation des brousses : repenser le foncier pastoral en Afrique de l'Ouest
}

Alexis Gonin

\section{To cite this version:}

Alexis Gonin. Les éleveurs face à la territorialisation des brousses: repenser le foncier pastoral en Afrique de l'Ouest. Annales de géographie, 2016, 2 (707), p. 28-50. 10.3917/ag.707.0028 . hal01327150

\section{HAL Id: hal-01327150 \\ https://hal.science/hal-01327150}

Submitted on 13 Dec 2017

HAL is a multi-disciplinary open access archive for the deposit and dissemination of scientific research documents, whether they are published or not. The documents may come from teaching and research institutions in France or abroad, or from public or private research centers.
L'archive ouverte pluridisciplinaire HAL, est destinée au dépôt et à la diffusion de documents scientifiques de niveau recherche, publiés ou non, émanant des établissements d'enseignement et de recherche français ou étrangers, des laboratoires publics ou privés. 


\section{Les éleveurs face à la territorialisation des brousses : repenser le foncier pastoral en Afrique de l'Ouest}

Herders coping with territorialization of space:

rethinking pastoral land tenure in West Africa

Alexis Gonin 


\section{Introduction}

Depuis une cinquantaine d'années, les systèmes d'élevage en Afrique de l'Ouest connaissent de profondes mutations. Le profil des éleveurs s'est diversifié au point où l'on rencontre tout un gradient de mobilités pastorales (des nomades aux sédentaires en passant par une grande variété de transhumants aux déplacements plus ou moins longs). D'un côté, les éleveurs sont plus ou moins engagés dans l'agriculture (pour les pasteurs, par exemple beaucoup de groupes touaregs, l'élevage demeure l'activité unique ; pour les agro-pasteurs, comme la majorité des groupes peuls, l'agriculture occupe une place importante dans l'économie familiale). D'un autre côté, les agriculteurs sont plus ou moins engagés dans l'élevage (on les appelle agro-éleveurs (Augusseau, 2004)). Si les pratiques et modes d'élevage se sont considérablement diversifiés, on constate une tendance générale à la diminution de la mobilité du cheptel (Turner et Hiernaux, 2008). Depuis les sécheresses des années 1970 et 1980, les cheptels nationaux ont considérablement augmenté (Inter-réseaux, 2015), faisant peser une pression plus grande sur les espaces de parcours. En effet, ces changements majeurs se sont réalisés dans des espaces ruraux eux-mêmes en pleine recomposition : le peuplement se densifie, ce qui alimente l'avancée des fronts pionniers agricoles qui réduisent les pâturages. Ces dynamiques sont à l'œuvre depuis la fin des années 1960, mais on arrive aujourd'hui, dans beaucoup de régions, proche d'un point de saturation foncière. II en résulte une exacerbation de la concurrence entre les mises en valeur agricole et pastorale des espaces ruraux. Face à ces dynamiques, les géographes, anthropologues, agronomes et zootechniciens se sont principalement intéressés à deux questions: l'intégration technique des activités agricoles et pastorales (amendement des champs par la fumure animale, culture attelée, pâturage des résidus de récolte... (Dugué et al. 2004, Vall et al. 2006, 2012) ; les conflits sociaux provoqués par une cohabitation souvent difficile entre les deux activités (Hagberg, 2001, Gautier, Ankogui-Mpoko, et al. 2005, Moritz, 2006). Depuis les travaux pionniers de Kintz (1982) sur le foncier pastoral puis ceux de Thébaud (2002), peu d'analyses ont été produites sur les conditions d'accès aux ressources pastorales. Pourtant, l'évolution des systèmes d'élevage et les recompositions spatiales des savanes changent radicalement le jeu foncier pour les éleveurs. Dans cet article, je me propose de montrer que l'on passe d'un régime foncier où la sécurisation foncière est fondée sur la réciprocité des droits d'accès à un régime où la sécurisation foncière est fondée sur l'appropriation exclusive des espaces de production. 
Un régime foncier peut être défini comme un ensemble de relations sociales, qui sont autant de rapports de pouvoir, dans lesquelles se négocie l'accès à la terre et aux ressources de l'environnement. Les règles d'accès sont dynamiques, elles peuvent être discutées, redéfinies (Barrière et Rochegude, 2008, Chauveau et al., 2001, Lavigne-Delville et Chauveau, 1998) ; par exemple les conflits d'accès aux ressources (entre agriculteurs et éleveurs, entre autochtones et migrants...) sont une occasion pour préciser, réaffirmer ou redéfinir les droits des acteurs en jeu (Le Meur et Hochet, 2010). Les régimes fonciers sont des structures socio-spatiales évolutives qui reflètent les représentations et pratiques de l'espace par une société. Les dynamiques des régimes fonciers sont donc un moteur de production de l'espace, au sens d'appropriation idéelle et matérielle des ressources par les groupes sociaux (Lefebvre, 1974). La transition d'un régime foncier pastoral à un autre est le symptôme du passage d'un mode de production de l'espace à un autre.

À chaque société, caractérisée à un moment de son histoire par un certain équilibre des rapports de pouvoir - équilibre qui transparaît dans l'architecture du régime foncier - correspond un type d'espace. Retaillé $(2005,2011)$ propose le concept d'espace mobile qui correspond assez bien au type d'espace produit par un régime foncier où les pasteurs dominent les rapports de pouvoir. Ce régime a été élaboré dans des zones peu densément peuplées, comme le Sahara et sa frange sahélienne. Mais lorsque les densités augmentent, que la concurrence pour l'accès aux ressources s'exacerbe et que les sédentaires dominent les rapports de force locaux, le principe fondamental des régimes fonciers change, supplanté par l'appropriation exclusive des ressources. C'est alors le concept de territorialisation, qui sera défini comme une stratégie spatiale de contrôle des ressources, qui traduit le mieux le processus de production de l'espace. Cet article vise à saisir le passage progressif de l'espace mobile à la territorialisation des espaces, c'est-à-dire le passage d'un processus de production de l'espace à un autre, par l'analyse des changements du régime foncier pastoral.

On n'analyse pas ici une recomposition par laquelle on passerait d'un état $A$ de départ à un état $B$ d'arrivée. Le terme de recomposition suppose un changement radical d'un état stable à un autre ; mais cette stabilité n'existe ni en amont, ni en aval du processus de changement (Richard, 2007). Notre point de départ, l'espace mobile, est un processus de production toujours recommencé. Le point d'arrivée (provisoire), la territorialisation des espaces, est un processus par essence toujours inachevé. L'espace mobile et la territorialisation sont dynamiques car produits par des relations sociales (vues ici dans leur dimension foncière) toujours mouvantes, toujours changeantes, " des matrices de transformation » (Foucault, 1976, p. 131).

Au-delà des aspects théoriques, les enjeux pour le développement sont importants. Le passage d'un régime foncier à un autre, et donc de l'espace mobile à la territorialisation des espaces, entrave la mobilité pastorale. Or, c'est grâce à la mobilité des troupeaux que les systèmes d'élevage ouest-africains sont relativement productifs, qu'ils assurent la résilience des sociétés d'éleveurs et qu'ils préservent les écosystèmes. L'analyse de l'évolution conjointe des régimes 
fonciers pastoraux et des types d'espaces qu'ils produisent est donc cruciale pour interroger le devenir de l'élevage en Afrique de l'Ouest.

\section{Régime foncier pastoral et production de l'espace mobile}

\subsection{Les mobilités : la meilleure réponse à l'incertitude pluviométrique}

L'Afrique de l'Ouest (mis à part la frange littorale) se caractérise par un régime pluviométrique uni-modal. La saison des pluies est de plus en plus courte et de moins en moins abondante à mesure que l'on se dirige vers le nord. La mousson ouest-africaine donne des précipitations très variables ; cette variabilité est d'autant plus grande que l'on se dirige vers le nord et les milieux sahéliens. Non seulement les totaux de précipitations peuvent connaître de grandes amplitudes d'une année à l'autre pour une région donnée, mais ces totaux peuvent être très différents au cours d'une même saison pour deux localités voisines de seulement quelques kilomètres. II n'y a pas non plus de régularité dans le rythme des précipitations d'une saison des pluies à l'autre : les pluies peuvent être en retard ou en avance en début de saison, s'arrêter plus tôt ou plus tard qu'attendu en fin de saison. La croissance et la sénescence de la végétation des pâturages sont très étroitement liées au régime pluviométrique. L'état des pâturages est donc lui aussi extrêmement variable dans le temps et dans l'espace en milieu sahélien. Face à cette géographie mouvante, la meilleure réponse que les pasteurs peuvent apporter pour maximiser les ressources et surmonter les contraintes est la mobilité des troupeaux (Behnke et al., 1993, Niamir-Fuller, 1999, Scoones, 1994). La mobilité présente trois avantages majeurs (Jullien, 2006) :

- Elle permet aux pasteurs d'adapter continuellement la charge animale aux capacités variables des pâturages. Longtemps accusées de dégradation des écosystèmes sahéliens et rendues responsables de la soi-disant désertification du Sahel (Milligan et Binns, 2007), les pratiques des pasteurs sont dans les faits respectueuses de l'environnement;

- Elle permet de maintenir une productivité animale supérieure à celle des troupeaux sédentaires dans des contextes similaires (Colin de Verdière, 1995) ;

- Enfin, elle renforce la résilience des pasteurs (Niamir-Fuller, 1998).

À l'échelle régionale, les pasteurs transhumants suivent des mouvements nordsud qui ont été abondamment décrits dans la littérature. De manière schématique, les troupeaux effectuent des transhumances entre d'une part des pâturages au nord où le fourrage est d'excellente qualité mais où la saison des pluies et donc la période végétative sont relativement courtes et d'autre part des pâturages plus au sud où il pleut plus, où il y a plus de fourrage, plus longtemps, mais où il y a plus de parasites durant la saison des pluies (Boureima et Boutrais, 2012, Boutrais, 1994, Brottem et al., 2014). Ce schéma régional englobe une succession de déplacements locaux de faible ampleur gérés au quotidien par les bergers pour s'adapter à l'état des pâturages et des points d'abreuvement, pour contourner les zones conflictuelles, pour profiter des opportunités offertes par 
les marchés... (Gonin et Tallet, 2012a, Marty et al., 2009, Welte, 1997). Durant les épisodes exceptionnels, comme les sécheresses de 1973-1974 et 1984-1985, les transhumances s'allongent vers le sud et les pasteurs restent plus longtemps dans les pâturages méridionaux (Gallais, 1976, 1984, Monod, 1975) A l'échelle locale comme à l'échelle régionale, dans les périodes normales comme dans les périodes exceptionnelles, la mobilité permet donc aux pasteurs de faire face à l'incertitude. Les groupes pastoraux sahéliens ont conçu un régime foncier adapté à ces pratiques qui leur garanti à la fois sécurité et flexibilité.

\subsection{Le régime foncier pastoral : la sécurisation par la protection des mobilités}

Dans les milieux semi-arides, l'eau est la ressource première, celle qui organise la mobilité des troupeaux. Un pâturage ne peut être exploité que s'il se trouve dans un rayon de 15 à 20 kilomètres autour d'un point d'eau. L'accès au point d'eau commande l'accès aux pâturages environnants et devient une clé de gestion des ressources pastorales (Thébaud, 2002). La famille qui a financé la construction d'un puits dans son terroir d'attache dispose, si l'on reprend la typologie proposée par Ostrom et Schlager (1992), des droits d'accès, de prélèvement et de gestion, mais pas de ceux d'exclusion ni d'aliénation.

Tab. 1 Les droits d'usage selon Ostrom et Schlager Land use rights according to Ostrom and Schlager

\begin{tabular}{|l|c|c|c|c|}
\hline & $\begin{array}{c}\text { Propriétaire } \\
\text { (owner) }\end{array}$ & $\begin{array}{c}\text { Possesseur } \\
\text { (proprietor) }\end{array}$ & $\begin{array}{c}\text { Ayant-droit } \\
\text { (claimant) }\end{array}$ & $\begin{array}{c}\text { Usager } \\
\text { autorisé } \\
\text { (authorized } \\
\text { user) }\end{array}$ \\
\hline $\begin{array}{l}\text { Accès et prélèvement } \\
\text { (access and withdra- } \\
\text { wal) }\end{array}$ & $\mathrm{X}$ & $\mathrm{X}$ & $\mathrm{X}$ & $\mathrm{X}$ \\
\hline $\begin{array}{l}\text { Gestion (manage- } \\
\text { ment) }\end{array}$ & $\mathrm{X}$ & $\mathrm{X}$ & $\mathrm{X}$ & \\
\hline $\begin{array}{l}\text { Exclusion } \\
\text { (exclusion) }\end{array}$ & $\mathrm{X}$ & $\mathrm{X}$ & & \\
\hline $\begin{array}{l}\text { Aliénation (aliena- } \\
\text { tion) }\end{array}$ & $\mathrm{X}$ & & & \\
\hline
\end{tabular}

Accès : droit d'entrer dans un espace ; Prélèvement : droit d'obtenir des produits d'une ressource (ex. herbe des pâturages) ; Gestion : droit de réguler les usages et d'améliorer la ressource par des infrastructures; Exclusion: droit de déterminer qui a un droit d'accès, et comment ce droit peut être transféré ; Aliénation : droit de vendre ou de louer l'un et/ou l'autre des droits d'exclusion et de gestion.

D'après Ostrom et Schlager, 1992.

Elle bénéficie d'un usage prioritaire du point d'eau : chaque jour, son troupeau est abreuvé en premier ; si le débit du puits est insuffisant, seul son troupeau est abreuvé. Mais cet usage prioritaire n'est pas un usage exclusif. Le troupeau d'une autre famille, des transhumants par exemple, a également un droit d'accès et de 
prélèvement de l'eau du puits. Ce droit est négocié. Il est généralement obtenu en échange de menus cadeaux (petites sommes d'argent, sucre, thé, veau, prêt de vaches...). Le gestionnaire du puits précise au visiteur le moment de la journée où il pourra abreuver son troupeau (généralement après le sien) et la durée maximale de son séjour. II est tacitement convenu que si le gestionnaire du puits part à son tour en transhumance, il pourra avoir accès au puits de son visiteur dans des conditions similaires. Premier avantage de ce régime foncier : les éleveurs se constituent un réseau social important qui ouvre largement le champ des destinations possibles de transhumance (Bonnet, 2014). Ils peuvent partir loin de chez eux à la recherche de pâturages de qualité en ayant la relative assurance de pouvoir en obtenir l'accès : leur mobilité est sécurisée. Deuxième avantage : les gestionnaires des puits adaptent sans cesse la charge animale à la capacité des pâturages environnants en jouant sur la durée du séjour de leurs visiteurs. Agronomes et zootechniciens ont donc pendant les années 1970 et 1980 assimilé à tort les pâturages sahéliens à des biens communs en accès libre et dont la dégradation, en suivant la théorie de la tragédie des communs de Hardin (1968), aurait découlé de l'absence de régulation ${ }^{1}$. L'étude approfondie des arrangements fonciers en milieu pastoral montre donc que cette théorie ne s'applique pas aux pâturages sahéliens. Troisième avantage du régime foncier pastoral : la fluidité des mobilités, et par conséquent la sécurité du mode de vie pastoral, est assurée à moindre coût. Il est en effet bien moins onéreux (en termes d'argent, de temps, de capital social et politique) de contrôler l'accès à des points (d'abreuvement) qu'à des surfaces (les pâturages). Le contrôle de surfaces nécessiterait une délimitation et une surveillance des limites tracées. Dans ce régime foncier pastoral, les limites territoriales sont délibérément floues et s'apparentent plus à des marges ou des confins, facilitant ainsi la fluidité des parcours de points d'abreuvement en points d'abreuvement (Thébaud, 2001).

\subsection{La production d'un espace mobile}

Pour Lefebvre (1974), le processus de production de l'espace passe par l'appropriation matérielle et idéelle des espaces et de ses ressources par les sociétés. La négociation des règles d'accès aux ressources et les pratiques qui en découlent participent pleinement de cette appropriation. Chaque société, caractérisée à un moment de son histoire par un certain équilibre des rapports de force et un certain mode de production, pour reprendre la terminologie marxiste de Lefebvre, produit un espace qui lui ressemble. Dans les régions politiquement dominées par les pasteurs, l'espace a été organisé par et pour la mobilité, ce dont témoigne le mode de gestion de l'accès aux puits et aux pâturages au Sahel, fondé sur la réciprocité. Les trois composantes des espaces produits décrites par Lefebvre

1 Selon cette théorie, les pâturages étant en accès libre, chaque pasteur pris individuellement aurait intérêt à maximiser son profit propre en faisant pâturer son troupeau sans se préoccuper de la dégradation générale du pâturage, dont les conséquences négatives ne sont pas supportées par lui seul mais par l'ensemble de la communauté (externalité négative). 
(espace vécu, espace perçu et espace conçu), ont longtemps été mal saisies par les géographes européens dont les concepts (territoires, limites...) sont inspirés par des points de vue de sédentaires. Retaillé (2005) propose le concept d'espace mobile pour mieux saisir ces trois composantes chez les populations sahariennes et sahéliennes. Si ces régions sont l'archétype de l'espace mobile, Retaillé (2011) défend l'idée que ce concept peut être élargi à d'autres espaces, en premier lieu l'espace mondial. Sans suivre l'auteur dans cette généralisation, force est de reconnaître que sa proposition théorique rend compte de manière efficace des processus de production de l'espace qui sont à l'œuvre dans les rapports fonciers au Sahel. Retaillé propose donc un renversement de nos représentations théoriques, en affirmant qu'au Sahara et au Sahel, le mouvement est premier, l'espace second. La mobilité ne s'organise pas selon des lieux et des territoires déjà donnés ; au contraire, les lieux surgissent, éphémères, de la mobilité des populations et de la fluidité des échanges sociaux; ils sont produits par les circonstances (Retaillé et Walther, 2013). Par exemple, un pâturage surgit de la conjonction des pluies tombées à tel endroit, du creusement d'un puits par une famille, de l'installation d'un campement et de l'arrivée de troupeaux. L'année suivante, ce lieu peut disparaître car cette conjonction d'événements ne sera pas arrivée à cet endroit. Les lieux et les routes apparaissent, disparaissent, se déplacent : l'espace mobile est par essence un espace fluide en continuelle construction.

II est important d'insister sur les conditions de production de l'espace mobile : rapports de force locaux dominés par les pasteurs ; faibles densités ; faible autorité des États et porosité de leurs cadres spatiaux (globalement les frontières et les territoires des États depuis l'époque coloniale n'entravent pas les circulations). Mais le régime foncier pastoral est aujourd'hui fragilisé, et la conception d'un espace mobile remise en question: certains points d'eau sont privatisés et l'accès aux pâturages environnants rendu exclusif ; les politiques étatiques et la décentralisation qui imposent leurs circonscriptions interfèrent négativement avec les pratiques de mobilité qui demeurent souvent mal comprises des décideurs ; les mobilités sont entravées partout où les parcours sont fragmentés par les espaces cultivés.

\section{Transformation des régimes fonciers et territorialisation des espaces}

\subsection{Dynamiques de l'interpénétration agriculture-élevage dans un contexte d'accroissement de la pression foncière}

Les élites politiques et administratives des colonies puis des pays indépendants, inspirées par certains travaux d'experts (Aldige, 1919, Doutressoulle, 1947) ont tenté d'imposer un espace régional des représentations où chaque zone bioclimatique aurait eu sa vocation de production. Ainsi l'élevage était vu comme l'apanage de la zone sahélienne, l'agriculture celui des zones soudanienne et soudano-guinéenne; au nord l'espace des nomades, au sud celui des sédentaires. 
Ces représentations de l'espace ont eu des conséquences importantes pour les populations. Ainsi, la loi $61-5$ du 25 mai 1961 au Niger fixe une limite au niveau du $15^{\mathrm{e}}$ parallèle : au sud de cette limite, la mise en valeur agricole est encouragée ; au nord elle est interdite (mis à part les champs de subsistance des pasteurs et les cultures dans les oasis). Ces représentations fixistes d'une réalité par essence mouvante sont très tôt critiquées. Les espaces de l'élevage et de l'agriculture s'étendent ou se rétractent au gré des variations pluviométriques interannuelles (Gallais, 1972, Retaillé, 2011) ou plus nettement encore lors des sécheresses (Gallais, 1976, Monod, 1975, Retaillé, 1989). L'interpénétration de l'élevage et de l'agriculture se joue à l'échelle locale et non pas le long d'un front régional qui traverserait toute l'Afrique de l'Ouest le long de sa bande sahélienne.

À l'échelle régionale, le glissement progressif du cheptel bovin sahélien des pasteurs peuls vers les zones soudanienne et soudano-guinéenne situées plus au sud (carte 1) contribue à brouiller un peu plus la représentation traditionnelle d'une partition zonale des activités (Boutrais, 1994, Bassett et Turner, 2007). Ces migrations ont commencé dès les années 1960 mais se sont accélérées après les sécheresses de 1973-1974 et 1984-1985. Elles ont été encouragées par l'éradication partielle de la trypanosomiase (une maladie mortelle pour les bovins, longtemps omniprésente dans les zones soudaniennes et soudano-guinéennes), le métissage des zébus avec des taurins pour les rendre résistants aux maladies, et les politiques accommodantes de certains pays côtiers comme la Côte d'Ivoire dans les années 1980 pour accueillir les pasteurs sahéliens sur leur territoire. Une autre représentation est mise en défaut : celle de la division pasteurs/agriculteurs. D'une part l'installation des pasteurs dans les zones soudanienne et soudano-guinéenne s'est en effet accompagnée d'une certaine diversification de leurs activités : ils ont été de plus en plus nombreux à pratiquer l'agriculture et à devenir ainsi des agro-pasteurs (d'Aquino, 2000). D'autre part, les observateurs attentifs notent dès les années 1970 un essor de l'agro-élevage, l'élevage pratiqué par les agriculteurs (Gallais, 1972). Les agriculteurs qui ont réussi à dégager une épargne monétaire grâce aux cultures de rente (coton, arachide, anacarde ${ }^{2} \ldots$..) l'ont investie dans l'élevage. Au fur et à mesure que la taille de leur troupeau augmentait, l'élevage s'est constitué comme le deuxième pilier économique de leur exploitation (Augusseau et al., 2004). Aujourd'hui, les agro-éleveurs détiennent une grande partie du cheptel dans les zones soudanienne et soudano-guinéenne ( $45 \%$ du cheptel bovin au Burkina Faso par exemple ${ }^{3}$ ).

Alors même que l'élevage connaissait ces profonds changements, les pâturages disponibles se sont considérablement réduits dans certaines régions sous l'effet de l'avancée de fronts pionniers agricoles (carte 1). L'augmentation des densités de population s'est accompagnée d'une extension des espaces cultivés au détriment des espaces de parcours. Pour ne donner que trois exemples, c'est le cas:

2 Le pédoncule de l'anacarde donne la noix de cajou

3 Source : Ministère des Ressources Animales, Ouagadougou, 2007. 


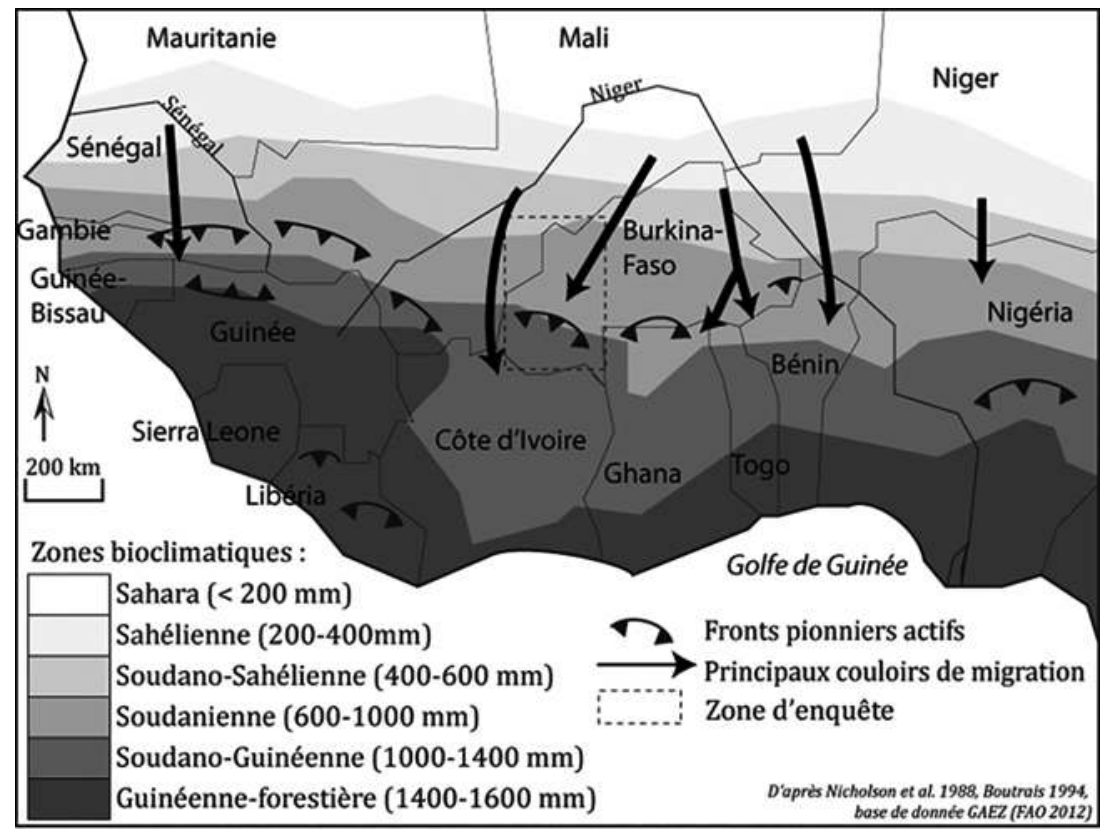

Fig. 1 Migrations pastorales et fronts pionniers agricoles en Afrique de l'Ouest Pastoral migrations and agricultural frontiers in West Africa

- de l'ouest du Burkina Faso, qui compte aujourd'hui 42 hab/km² contre moins de $10 \mathrm{hab} / \mathrm{km}^{2}$ en 1968 (Savonnet, 1968);

- du nord du Togo, où la densité de la région des Savanes est passée de 38 à $90 \mathrm{hab} / \mathrm{km}^{2}$ entre 1981 et 2010 , de 37 à $62 \mathrm{hab} / \mathrm{km}^{2}$ pour la région de Kara ${ }^{4}$;

- de la Haute-Casamance au Sénégal où les densités étaient de $40 \mathrm{hab} / \mathrm{km}^{2}$ en 2002 contre $20 \mathrm{hab} / \mathrm{km}^{2}$ en $1988^{5}$.

Les fronts pionniers des années 1960-1970 se situent à proximité immédiate de régions historiquement densément peuplées. C'est le cas de la marge ouest du plateau mossi au Burkina Faso (Kohler, 1972) ou de la plaine sableuse du seeno en contrebas de la falaise de Bandiagara en pays dogon au Mali (Thibaud, 2005). Les régions qui connaissent les plus fortes augmentations de densités depuis les années 1980 se situent plutôt dans la zone soudanienne (Fanchette, 2011, p. 55). Historiquement moins peuplées, elles accueillent des migrants agricoles en provenance des noyaux de hautes densités (cas des migrations des agriculteurs du bassin arachidier vers la Haute-Casamance au Sénégal ou de ceux du plateau mossi vers l'ouest du Burkina Faso et la Côte d'Ivoire). Si beaucoup de ces régions ont d'ores et déjà atteint de hauts niveaux de densités et sont

4 Bureau central du recensement, Togo, 2011.

5 Agence Nationale pour la Statistique et la Démographie (ANSD), Sénégal 
désormais des zones de départ (le Sud Mali, l'Ouest burkinabè, le Sud-Niger, le Nord-Nigéria, le Nord-Cameroun), d'autres enregistrent à l'heure actuelle des densités encore relativement basses mais connaissent des dynamiques de peuplement accélérées sur la dernière décennie. La conséquence à l'échelle locale de ces dynamiques régionales est l'accroissement de la concurrence entre usagers pour l'accès aux derniers espaces non cultivés disponibles (désignés localement par le terme générique de brousse), vues par les agriculteurs comme des réserves de terres cultivables, et par les éleveurs comme des pâturages.

\subsection{Concurrences, rapports de pouvoir, territorialisation}

Les producteurs locaux ont conscience des dynamiques d'avancée des champs et de disparition des brousses. Pour pouvoir nourrir leur famille, qui ne cesse de s'agrandir, et transmettre un espace de production suffisant à leurs enfants dans un contexte de compétition accrue pour l'accès aux ressources, ils cherchent à s'approprier localement des portions de brousses. Comme l'ont montré Ostrom et Schlager (1992), l'appropriation (property) de biens communs ne se réduit pas à la propriété privée (ownership). Toutefois, en situation de concurrence croissante et face au sentiment d'insécurité qu'alimente la saturation progressive des espaces de production, les acteurs cherchent à consolider leurs droits d'accès, de prélèvement et de gestion par un droit d'exclusion (figure 2). Dans les régions où les espaces se ferment sous l'effet conjoint de la diminution des ressources et de l'augmentation du nombre des usagers, l'acquisition du droit d'exclusion semble aux acteurs le moyen le plus à même d'assurer leur sécurisation.

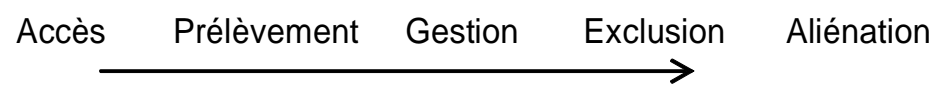

Gagnants des processus de territorialisation

D'après Ostrom et Schlager, 1992

Fig. 2 Territorialisation et dynamique des droits d'usages Territorialization and dynamics of land use rights

Les effets de cette dynamique sociale sur les espaces peuvent être appréhendés par le concept de territorialisation. En s'inspirant de $\operatorname{Sack}^{6}(1983,1986)$, Vandergeest et Peluso ${ }^{7}$ (1995) et Peluso ${ }^{8}$ (2005) on peut définir la territorialisation comme une stratégie spatiale déployée par des acteurs (individus, institutions, Etat) engagés dans des rapports de pouvoir pour contrôler l'accès à une ressource

6 «Territoriality is a spatial strategy to affect, influence, or control resources and people, by controlling area" $(1986,5)$

7 "Territorialization is about excluding or including people within particular geographic boundaries, and about controlling what people do and their access to natural resources within those boundaries » (388)

8 Territorialization is « the creation and maintenance of spatialized zones within certain practices are permitted based on the explicit or implicit allocation of rights, controls, and authority » 
par le dessin d'une aire géographique délimitée sur laquelle ces acteurs cherchent à faire reconnaître leur autorité. La territorialisation est une stratégie spatiale dans le sens où les acteurs utilisent et transforment un espace en moyen de contrôle des ressources. « Le territoire est généré à partir de l'espace, il est le résultat d'une action conduite par un acteur syntagmatique (acteur réalisant un programme) à quelque niveau que ce soit. En s'appropriant concrètement ou abstraitement (par exemple, par la représentation) un espace, l'acteur « territorialise » l'espace. » (Raffestin 1980, p. 129). Selon Raffestin, les acteurs projettent leurs représentations sur l'espace et mènent à bien leur projet en traçant des limites et en cherchant à faire reconnaître un pouvoir, des règles, une autorité. Les actions spatiales produisent des nœuds, des réseaux et des maillages qui constituent l'armature des nouveaux territoires et « permettent d'assurer le contrôle sur ce qui peut être distribué, alloué, et/ou possédé » (p. 136).

La délimitation est une action particulièrement importante dans la genèse d'un territoire. Les limites spatiales sont les limites d'extension du pouvoir qui s'affirme sur les ressources. Elles participent donc de la définition de ce pouvoir. Les limites spatiales, si elles sont claires et visibles par tous, permettent de signifier, de communiquer le pouvoir. Elles le rendent ainsi effectif, exécutable, voire contestable. Les limites peuvent prendre une variété de formes matérielles: marques sur l'écorce des arbres, série de rochers, cours d'eau, fil barbelé, limites d'une défriche, points relevés au GPS et consignés dans un document de type cadastral... Mais la délimitation n'est pas le seul moyen de mener une territorialisation. Peluso (2005) critique l'approche de Sack (1983) en arguant que la territorialisation n'est pas toujours aussi explicite ou intentionnelle que celui-ci le laisse entendre: "Les changements dans les pratiques quotidiennes peuvent être aussi effectifs que les stratégies ouvertes et directes pour revendiquer des droits sur les ressources territoriales. Les mots et les actions, comme les cartes, comptent » (2005, p. 4). Fréquenter quotidiennement un même pâturage, couper les branches de certains arbres pour le fourrage (Gautier, Bonnerat, et al. 2005), ramasser les fruits de certains arbres, défricher une brousse, causer des dégâts intentionnels dans un champ cultivé, allumer un feu de brousse, édicter des interdits coutumiers, faire des sacrifices, proférer des menaces... sont autant d'actions spatiales qui peuvent entrer dans le cadre d'une stratégie de territorialisation. Par ailleurs, la territorialisation permet d'inclure certains acteurs, d'en exclure d'autres, de proscrire certaines activités, d'en prescrire d'autres (Vandergeest et Peluso, 1995) : ce faisant elle participe à la définition des ressources. Par exemple, une brousse, composée d'une forêt claire traversée par un marigot est à la fois une réserve de gibier, une source de production de produits forestiers ligneux et non ligneux, un ensemble de terres fertiles, un pâturage. Tous ces usages potentiels ne sont pas compatibles entre eux. Selon que les chasseurs, les cueilleurs, les bûcherons, les agriculteurs ou les éleveurs mèneront un processus de territorialisation, c'est l'un ou l'autre ou plusieurs de ces usages qui seront privilégiés au détriment d'autres. La brousse sera alors une ressource fourragère, et/ou cynégétique, ou agricole... Appropriation d'un 
espace comme résultante des pratiques des acteurs et des jeux de pouvoir auxquels ils participent, "le territoire est une production de l'espace " (Raffestin, 1980,

p. 130) et la territorialisation est le processus correspondant. L'analyse de cas concrets d'appropriation des ressources observés sur le terrain permettra de dévoiler quelques-uns des mécanismes de territorialisation caractéristiques d'un changement de régime foncier et du passage d'un mode de production de l'espace à un autre.

\subsection{Les éleveurs face à la territorialisation}

Les processus de territorialisation compliquent la donne pour les éleveurs car ils fragmentent l'espace, introduisent de la discontinuité et entravent les mobilités. Quatre exemples issus d'un terrain de recherche dans l'ouest du Burkina Faso (encadré 1, carte 2) montrent en quoi l'évolution des régimes fonciers participe à la territorialisation des espaces et illustrent les difficultés que ce processus oppose aux éleveurs.

\section{RÉGION D'ÉTUDE ET MÉTHODOLOGIE D'ENQUÊTE}

L'ouest du Burkina Faso est une région située en majeure partie dans la zone soudanienne. Longtemps considéré, dans les plans nationaux d'aménagement, comme le grenier agricole du pays et bassin historique de la production cotonnière, l'Ouest est aussi, depuis les années 1980, une grande région d'élevage. Toutefois, la croissance démographique soutenue $\left(+2,6 \% /\right.$ an entre 1985 et $\left.2006^{\circ}\right)$ et les politiques de développement ont alimenté l'avancée d'un front pionnier agricole et une contraction des pâturages, situation exemplaire de la recomposition de beaucoup d'espaces ruraux au cours des cinquante dernières années. Le résultat est l'accroissement de la compétition foncière entre usagers à l'échelle locale. En 2012 et 2013, deux séjours sur le terrain ont été l'occasion d'avoir des entretiens avec 165 producteurs (agro-pasteurs, agro-éleveurs et agriculteurs), 59 personnes-ressources (responsables politiques, coutumiers, associatifs, experts et membres d'ONG) ; en outre 24 entretiens collectifs (dont 14 cartographies participatives) ont été animés dans les villages de trois communes d'enquêtes (carte). L'objectif était de recueillir des données sur la mobilité des troupeaux, le recul des espaces de parcours, et les enjeux et rapports de pouvoirs locaux et nationaux autour de l'accès aux ressources pastorales (Gonin, 2014). Dans cette partie, seuls quatre exemples illustratifs des processus de territorialisation sont présentés.

\subsubsection{La territorialisation des résidus de récolte}

La vaine pâture est le droit pour tous les éleveurs d'exploiter les résidus de culture (tiges de maïs, mil, sorgho, coton, fanes d'arachides...) après que les récoltes ont été effectuées. Elle entre dans le cadre d'un contrat tacite bien décrit dans la littérature (Benoit, 1982, Bonfiglioli, 1990) qui organise l'échange d'amendement des champs par la fumure animale contre les résidus, un fourrage de bonne qualité. Les agro-pasteurs cultivent de petites surfaces, et ne bénéficient, pour leur propre compte, que d'une quantité de résidus assez limitée. C'est pour

9 Source : Institut National de la Démographie et de la Statistique, 1985, 2006. 

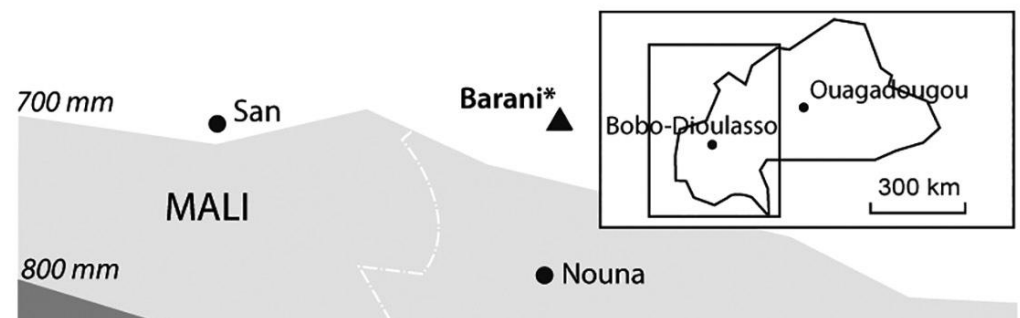

Dédougou

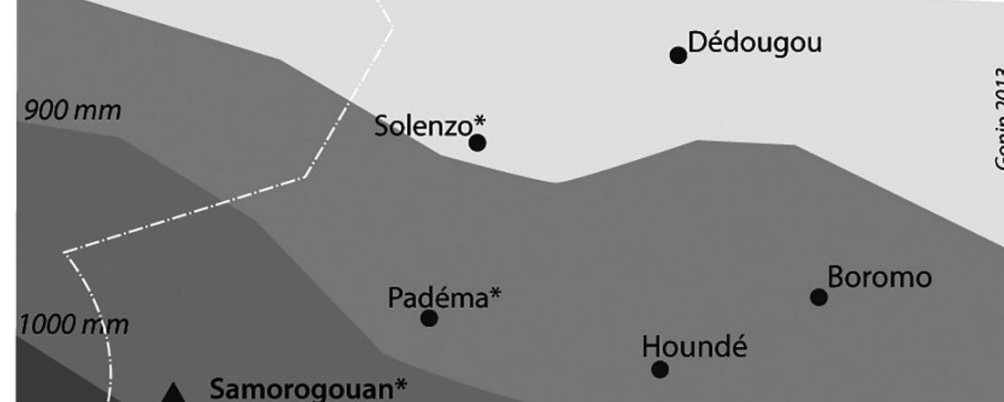

Samorogouan*

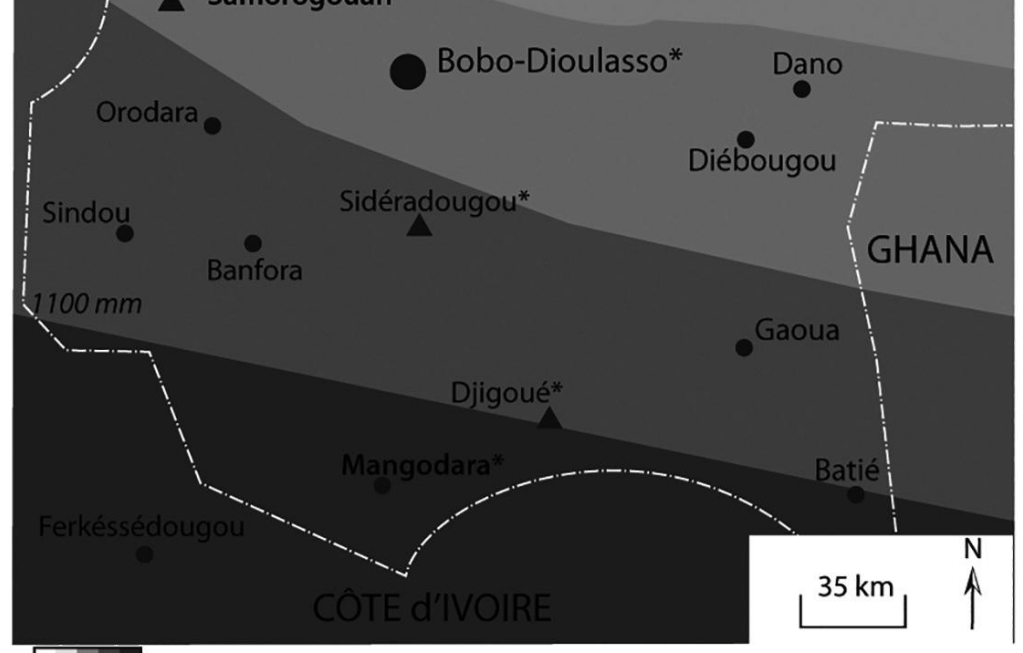

I. Isohyètes (700-1100 mm)

Barani* Commune où les principales enquêtes de terrain ont été menées

Djigoué* Autre commune d'enquête

- Aire pastorale aménagée

Fig. 3 Localisation des zones de recherche de terrain Location of study areas 
cette raison qu'ils ont l'habitude, dès la fin des récoltes pendant la saison sèche et froide (décembre-janvier), d'aller faire paître leurs animaux dans les champs des agriculteurs. Or avec le développement de l'agro-élevage, la concurrence est de plus en plus forte sur ces résidus. Comme l'observent également Augusseau (2007) et Breuser (1998) dans l'ouest du Burkina et Dongmo et al. (2012) dans l'ouest du Burkina et dans le Nord Cameroun, les agro-éleveurs ont tendance à réserver l'accès aux résidus de leurs champs à leur troupeau tandis que les autres éleveurs sont exclus de ressources auparavant ouvertes à tous. On peut évoquer une territorialisation des pâturages de résidus de récolte durant la saison sèche et froide. Les champs peuvent être considérés comme des espaces délimités (chaque agriculteur connaît la limite de son champ, ainsi que les gestionnaires fonciers). Les enquêtes de terrain montrent que les agro-éleveurs propriétaires des champs cherchent à s'arroger un droit d'exclusion. Ils affirment être les seuls autorisés à utiliser les résidus de leurs cultures ; à ceux qui viennent leur demander un droit d'accès et de prélèvement, ils le refusent; quand cela est possible, ils placent un enfant pour surveiller qu'aucun autre troupeau ne pénètre dans les champs où se trouvent les résidus. Ce contrôle ne peut toutefois pas être totalement effectif, la surveillance des résidus étant trop coûteuse en temps et en main-d'œuvre. II y a donc bien un effort de territorialisation de ces ressources, même s'il demeure inabouti et qu'il ne concerne pas la totalité des champs au sein d'un même terroir.

\subsubsection{Défrichements stratégiques et territorialisation des pâturages de saison des pluies}

Dans les terroirs villageois, les défrichements des champs dans la brousse ne s'effectuent pas de manière concentrique autour du village, ce qui serait la logique spatiale la plus appropriée pour réduire les trajets entre les lieux d'habitation et les lieux de culture. Les champs sont défrichés selon les pointillés de lignes imaginaires qui entourent des brousses revendiquées par les lignages autochtones détenteurs des droits fonciers coutumiers. Les défriches permettent de rendre visibles, par la mise en valeur agricole, les limites revendiquées des maîtrises foncières des différents lignages autochtones d'un village donné. Le chef de village de Djingouan (commune de Samorogouan, dans l'ouest du Burkina Faso) témoigne : "Quand les migrants s'installent, est-ce que vous leur montrez une limite qu'ils ne doivent pas dépasser avec leur champ ? Oui. On leur montre des limites naturelles. Ceux qui sont venus en 1992 [hameau mossi de Koko dépendant du village de Djingouan], on leur a montré la grande route, qui croise un marigot. On leur a dit vous descendez ce marigot. II y a un sentier qui va à Sibougou. On leur a dit c'est cette portion-là » (2 mai 2013). L'espace de la brousse est délimité par les éléments naturels et les voies de passage indiqués par le chef. En installant des migrants et en leur accordant des droits d'accès, de prélèvement (les récoltes) et de gestion (les migrants peuvent installer de nouveaux arrivants en en référant au chef de village, ils peuvent investir pour restaurer la fertilité du sol...), les autochtones réaffirment leur droit d'exclusion sur cette brousse (ils accordent la terre à telle famille de migrants, mais pas à telle autre ; ils délèguent leur droit de gestion). 
La défriche stratégique des brousses pour l'agriculture entre dans le cadre d'un processus de territorialisation par le contrôle de l'accès aux ressources d'un espace au moyen de la délimitation d'une aire géographique sur laquelle les autochtones font reconnaître leur autorité. De fait, cette territorialisation exclut pendant toute la durée de la campagne agricole (de juin à novembre) les éleveurs d'une brousse qu'ils utilisaient comme pâturage de saison des pluies. Dans les terroirs où les brousses ont été massivement défrichées et cultivées, il reste toutefois quelques résidus de pâturages de saison des pluies, généralement situés sur des collines incultes. Les enquêtes dans les villages de l'Ouest burkinabè montrent alors qu'à taille de troupeau égale, ce sont les bovins des agro-pasteurs qui doivent partir hors du terroir vers des zones éloignées où il reste des pâturages plus importants, tandis que les troupeaux des agro-éleveurs restent sur place. II y a là une incitation, implicite ou explicite, faite aux agro-pasteurs de laisser le bénéfice des pâturages locaux aux agro-éleveurs du village. La territorialisation des derniers pâturages de saison des pluies dans les terroirs villageois se fait donc plutôt au détriment des agro-pasteurs.

\subsubsection{Plantation d'anacardiers et territorialisation des pâturages de saison sèche}

Dans la zone soudano-guinéenne, on assiste à des logiques semblables de territorialisation des brousses dans les bassins de production de l'anacarde ${ }^{10}$ (Audouin et Gonin, 2014). Le fait de planter un anacardier est un moyen d'affirmer des droits forts sur une terre : accès, prélèvement, gestion, exclusion, voire aliénation. Les plantations ne sont pas le fruit du hasard : elles participent à une stratégie spatiale d'appropriation de la terre à des fins exclusives sur des zones de front pionnier agricole. Durant la période des récoltes, de février à avril, les troupeaux bovins sont chassés des vergers car ils ingèrent les fruits. Dans ce cas, la territorialisation exclut les éleveurs des pâturages de saison sèche qui étaient leur destination de transhumance. En saison des pluies, les animaux peuvent de nouveau entrer dans les vergers et pâturer sous les arbres. Toutefois, les planteurs clôturent de plus en plus leurs vergers, pour les protéger des bovins durant les récoltes. Les clôtures restent en place durant la saison humide et changent alors de fonction : elles permettent de réserver l'accès au verger devenu pâturage aux animaux que le planteur a acquis grâce aux revenus de l'anacarde.

\subsubsection{Une territorialisation par et pour les éleveurs}

Le village de Barani fait exception au sein des savanes soudaniennes car la territorialisation des brousses s'y réalise en faveur des agro-pasteurs et de la pratique du pastoralisme. Le village de Barani est la capitale d'un ancien royaume peul, très puissant localement jusqu'à la colonisation française à la fin du XIX ${ }^{\mathrm{e}}$ siècle. Néanmoins, à partir du milieu du $\mathrm{xx}^{\mathrm{e}}$ siècle, l'influence politique et économique de Barani décroît, les agro-pasteurs peuls Sidibe de Barani émigrent vers le Mali et le Nord de la Côte d'Ivoire, et leurs anciens esclaves, les rimaybe, gagnent en

10 L'anacarde donne la noix de cajou. 
influence. Une grande plaine sableuse sert historiquement de pâturage de saison des pluies, tandis qu'en saison sèche, les troupeaux partent en transhumance dans les bourgoutières (pâturages de plaine inondable) du Sourou (carte 3). Avec le déclin de la chefferie de Barani, des agriculteurs installent leurs champs dans le grand pâturage de saison des pluies. Au début des années 1990, l'arrivée d'un projet de développement, le PDRI (Projet de Développement Rural Intégré), financé par l'Agence Française de Développement (AFD) va changer la donne spatiale et politique. Après concertation avec les populations locales, les 50000 ha de pâturage de saison des pluies sont délimités, bornés avec des balises en béton ; un cahier des charges de la nouvelle zone pastorale est établi, fixant les règles d'utilisation; la zone est exclusivement réservée à l'élevage et les agriculteurs installés en sont chassés; des comités de surveillance font respecter le cahier des charges et perçoivent les droits d'entrée payés par les éleveurs qui veulent utiliser le pâturage. La zone pastorale de Barani est l'exemple exceptionnel d'une territorialisation aboutie en faveur de l'élevage dans une zone de forte pression agricole. Elle est bien le résultat d'une stratégie spatiale pour contrôler l'accès et l'usage d'une brousse au moyen d'une aire géographique que les agropasteurs peuls Sidibe, avec l'aide du PDRI, ont délimité et sur laquelle ils ont fait reconnaître leur autorité.

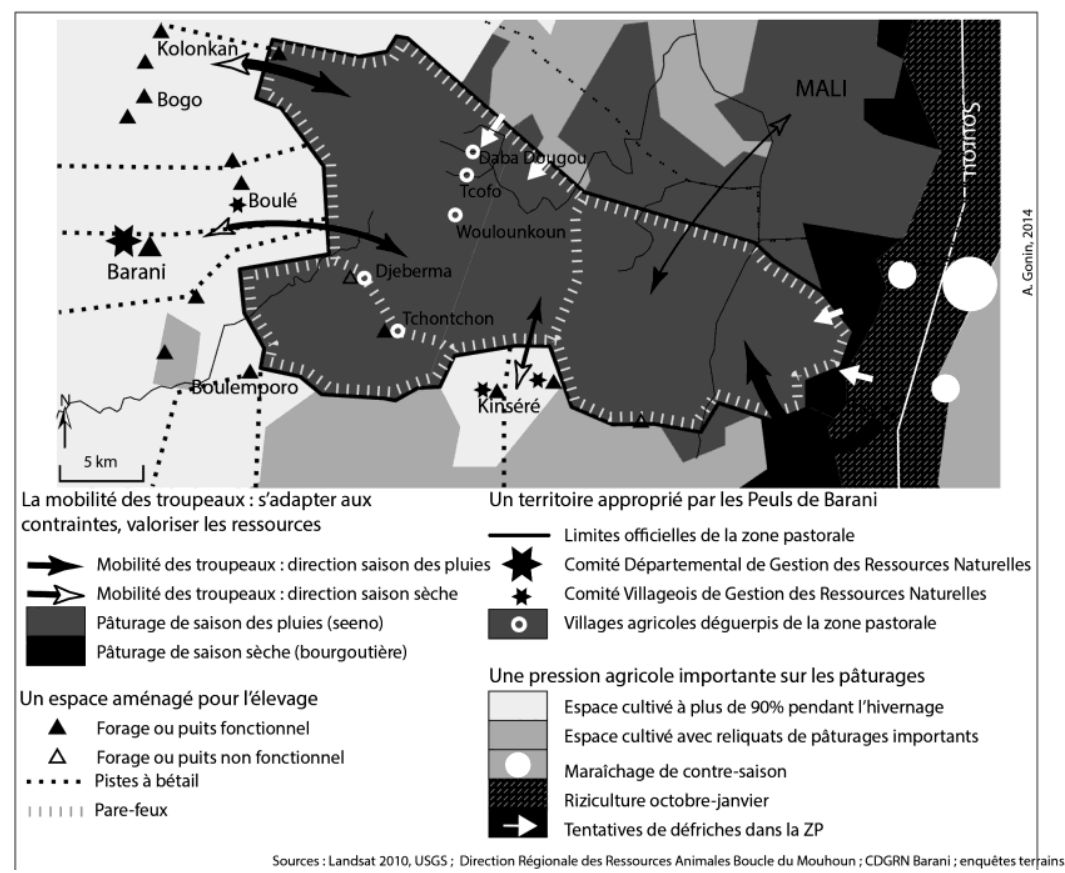

Fig. 4 Territorialisation des espaces de parcours à Barani Territorialization and rangelands in Barani 


\subsubsection{Les éleveurs, perdants de la territorialisation ?}

Mis à part l'exemple de Barani qui demeure un cas exceptionnel, la territorialisation des brousses se réalise généralement en défaveur des éleveurs. Ils voient le volume du fourrage disponible diminuer, en saison des pluies, avec la réduction de l'étendue des pâturages, au début de la saison sèche avec la remise en cause de la vaine pâture et en deuxième partie de saison sèche avec l'extension des vergers d'anacardiers dans les pâturages qui sont la destination des transhumances. Les parcours sont rendus plus difficiles avec la fragmentation des espaces. Les conflits avec les agriculteurs qui ont pour origine les dégâts aux cultures causés par les animaux ayant échappé à la surveillance de leur berger se multiplient dans les zones proches de la saturation agricole et dans les bassins anacardiers. La mise en culture des pistes à bétail entrave les mobilités à l'échelle locale comme à l'échelle régionale lors des grandes transhumances nord-sud. Enfin, les inégalités s'accroissent entre, d'une part, les agro-pasteurs les plus pauvres en capital social, politique et économique qui perdent l'accès aux ressources pastorales et, d'autre part, les agro-éleveurs qui territorialisent les derniers pâturages locaux à leur profit et les agro-pasteurs les plus riches qui ont les moyens de continuer leur migration vers des zones qui ne sont pas encore atteintes par les fronts pionniers agricoles (Gonin, 2014).

\section{Discussion : de l'espace mobile à la territorialisation de l'espace}

\subsection{Cristallisations territoriales aux échelles locale et régionale}

L'accroissement de la pression foncière dans certaines régions ouest-africaines s'est accompagné d'une transition d'un régime foncier pastoral fondé sur une certaine réciprocité dans l'accès aux ressources vers des régimes fonciers fondés sur une appropriation plus ou moins exclusive des ressources. Cette évolution des régimes fonciers dénote un changement plus large dans les logiques de production de l'espace. Le tableau 2 vise à synthétiser les implications pratiques et théoriques de ces mutations foncières et spatiales.

La conceptualisation qui appréhende le mieux l'organisation des espaces peu densément peuplés semble être celle de l'espace mobile. Au cours de leur migration vers le sud, les pasteurs sahéliens ont emporté avec eux leur représentation de l'espace et le régime foncier qui lui était lié. Toutefois, à mesure que les densités de population augmentent, que les brousses sont défrichées et cultivées et que les pâturages diminuent, la concurrence pour l'accès aux ressources s'accroît. La territorialisation est enclenchée et fait perdre de sa fluidité à l'espace mobile. Celui-ci doit se mouler dans les mailles de plus en plus rigides des espaces denses (en termes de peuplement et d'occupation de l'espace). La brousse est découpée, délimitée, appropriée : bien que ces actions demeurent inachevées, l'espace se cristallise peu à peu en territoires. Les marges de manœuvre spatiales se réduisent. Les lieux éphémères qui sont les points d'achoppement provisoires des 
Tab. 2 Transformation des régimes fonciers et des modes de production de l'espace Transformation of land tenure systems and methods of production of space

\begin{tabular}{|c|c|c|c|}
\hline & & $\begin{array}{l}\text { Espace mobile et } \\
\text { régimes fonciers fondés } \\
\text { sur la réciprocité des } \\
\text { droits d'accès }\end{array}$ & $\begin{array}{l}\text { Vers la territorialisation } \\
\text { et une appropriation } \\
\text { exclusive des } \\
\text { ressources }\end{array}$ \\
\hline \multirow{4}{*}{ 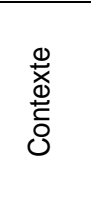 } & Densités de population & Faibles & En forte augmentation \\
\hline & Densités du bétail & Faibles & En forte augmentation \\
\hline & Champs & Ponctuels & $\begin{array}{l}\text { Tous les espaces fertiles } \\
\text { tendent à être mis en } \\
\text { culture }\end{array}$ \\
\hline & $\begin{array}{l}\text { Variabilité des ressources } \\
\text { dans le temps et l'espace }\end{array}$ & Très forte & Forte \\
\hline \multirow{3}{*}{ 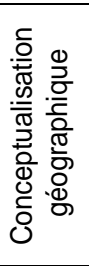 } & $\begin{array}{l}\text { Processus de production de } \\
\text { l'espace }\end{array}$ & Espace mobile & $\begin{array}{l}\text { Territorialisation } \\
\text { l'espace }\end{array}$ \\
\hline & $\begin{array}{l}\text { Structures spatiales de base } \\
\text { du régime foncier }\end{array}$ & $\begin{array}{ll}\text { Points } & \text { (d'abreuvement) } \\
\text { réseaux } & \text { (parcours des } \\
\text { troupeaux) } & \end{array}$ & $\begin{array}{l}\text { Mailles (pâturages appro- } \\
\text { priés) ; réseaux (parcours) }\end{array}$ \\
\hline & Limites spatiales & $\begin{array}{ll}\text { Délibérément floues; } & \text { marges, confins. }\end{array}$ & $\begin{array}{l}\text { Souvent implicites, parfois } \\
\text { explicitement marquées; } \\
\text { dans tous les cas imposées } \\
\text { aux éleveurs }\end{array}$ \\
\hline \multirow{7}{*}{ 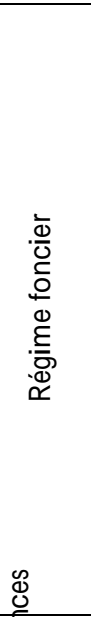 } & $\begin{array}{l}\text { Mise en valeur des espaces } \\
\text { la mieux reconnue sociale- } \\
\text { ment et politiquement }\end{array}$ & Pastoralisme & Agriculture \\
\hline & $\begin{array}{l}\text { Clé de gestion des res- } \\
\text { sources pastorales }\end{array}$ & Accès aux puits & $\begin{array}{l}\text { Autorité qui cherche à } \\
\text { imposer son contrôle sur } \\
\text { une ressource }\end{array}$ \\
\hline & Acteurs des négociations & $\begin{array}{l}\text { Pasteurs et agro-pasteurs } \\
\text { d'un même réseau } \\
\text { communautaire }\end{array}$ & $\begin{array}{l}\text { Agro-pasteurs et agro- } \\
\text { éleveurs. Acteurs aux } \\
\text { logiques et objectifs socio- } \\
\text { économiques différents. }\end{array}$ \\
\hline & Rapports de force locaux & Pasteursdominants & $\begin{array}{l}\text { Agro-éleveurs sédentaires } \\
\text { dominants }\end{array}$ \\
\hline & $\begin{array}{l}\text { Droit sécurisant que } \\
\text { les acteurs cherchent à } \\
\text { atteindre }\end{array}$ & Réciprocité & Exclusivité \\
\hline & Principe de sécurisation & Mobilité & $\begin{array}{l}\text { Appropriation à tendance } \\
\text { exclusive }\end{array}$ \\
\hline & $\begin{array}{l}\text { Droits du gestionnaire des } \\
\text { ressources }\end{array}$ & Accès, prélèvement, gestion & $\begin{array}{ll}\text { Accès, } & \text { prélèvement, } \\
\text { gestion, et } & \text { construction } \\
\text { progressive d'un droit } \\
\text { d'exclusion }\end{array}$ \\
\hline \multirow{2}{*}{ 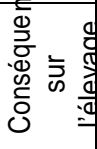 } & Mobilités & $\begin{array}{l}\text { Régime foncier conçu pour } \\
\text { favoriser les mobilités }\end{array}$ & $\begin{array}{l}\text { Mobilités peu à peu entra- } \\
\text { vées. Accroissement des } \\
\text { inégalités entre éleveurs. }\end{array}$ \\
\hline & $\begin{array}{l}\text { Résilience du système d'éle- } \\
\text { vage }\end{array}$ & Forte & En voie de fragilisation \\
\hline
\end{tabular}

réseaux de mobilité s'ancrent et se fixent ; l'écheveau des routes se réduit à une seule piste, souvent obstruée par les champs. L'idée de gradient est essentielle : il n'y a pas de rupture mais des déclinaisons dans l'avancée et les modalités de la territorialisation. À l'échelle locale, la cristallisation territoriale est souvent inaboutie. Les tentatives de délimitation et de contrôle de l'espace demeurent inachevées, résultats de rapports de force qui ne parviennent jamais à un équilibre définitif. Si l'espace a alors perdu de sa fluidité, il n'est pas non plus franchement territorialisé. On doit donc faire preuve d'un grand sens de la nuance pour rendre 
compte de l'articulation entre espace mobile et territorialisation de l'espace. Les deux modes de production de l'espace se superposent plus souvent qu'ils ne se juxtaposent.

À l'échelle régionale, on peut tenter une généralisation qui donne à voir les dynamiques des deux modes de production de l'espace. Autour de noyaux où l'on observe des cristallisations territoriales, l'espace demeure mobile. Ainsi, si l'espace mobile est le mode principal au Sahara et dans la bande sahélienne, régions de faibles et très faibles densités, la territorialisation de l'espace prend de l'ampleur dans les régions où les densités rurales augmentent fortement. L'espace mobile est toutefois encore le mode dominant des campagnes soudaniennes et soudanoguinéennes qui n'ont pas encore été touchées par l'avancée des fronts pionniers. À l'échelle régionale, la répartition espace mobile/territorialisation de l'espace ne correspond pas aux divisions zonales. Une image des régimes fonciers et du mode de production de l'espace en Afrique de l'Ouest serait celle d'une peau de léopard dont la meilleure approche serait les cartes de densités ${ }^{11}$ de population et celles de degré de mise en valeur agricole ${ }^{12}$. Les tâches correspondraient aux noyaux où la territorialisation de l'espace est enclenchée. Entre ces tâches, l'espace demeurerait mobile. La trame des tâches est plus lâche dans la zone sahélienne. Les taches ont tendance à s'étendre, mais si l'on analyse cette diffusion de plus près, on constate que la bordure des tâches n'est pas nette. Là où la territorialisation de l'espace s'étend, l'espace mobile transparaît encore. De retour à l'échelle régionale, cela donne l'impression d'une peau de léopard délavée.

Prenant acte de cette articulation des espaces à l'échelle régionale, le défi aujourd'hui pour les éleveurs et les décideurs politiques est de faire reconnaître des droits fonciers préservant d'une part la fluidité des espaces encore mobiles et, d'autre part, adaptés aux espaces en cours de territorialisation.

\subsection{Production de l'espace et sécurisation foncière des éleveurs}

La lecture des évolutions des régimes fonciers par le prisme du couple espace mobile/territorialisation de l'espace permet de mettre en lumière les menaces qui pèsent sur l'élevage en Afrique de l'Ouest à travers les obstacles opposés aux mobilités pastorales. Elle incite aussi à penser d'autres rapports possibles des éleveurs à l'espace, adaptés au contexte. Les pasteurs pratiquent en effet les deux types d'espace. Le régime foncier pastoral fondé sur la réciprocité sécurise les pasteurs dans les espaces mobiles. Les éleveurs y sont en position de dominante dans les rapports de force locaux. Les avancées législatives récentes dans certains pays comme le Niger (ordonnance 2010-029 relative au pastoralisme) protègent dans une certaine mesure la fluidité de l'espace mobile. Mais les pasteurs rencontrent plus de difficultés dans les espaces soumis au processus de territorialisation. La fragmentation des parcours fragilise les mobilités. II faut donc penser autrement le rapport des éleveurs à l'espace dans ces régions. Il est

11 www.worldpop.org.uk consulté le 8 juin 2015.

12 gaez.fao.org consulté le 8 juin 2015. 
possible d'y concevoir une territorialisation qui promeut à la fois la sécurisation foncière des éleveurs et celle des agriculteurs. Les axes de circulation et les aires pastorales seraient dans cette logique délimités et protégés par des règles d'usage légitimes et des comités de régulation composés de différents types d'usagers. Des opérations de délimitation de pistes à bétail menées au Niger (Moutari et Giraut, 2013), au Nord-Cameroun (Moritz et al., 2013), dans l'Ouest burkinabè (Gonin et Tallet, 2012b) ou à l'est du Sénégal (Kitchell et al., 2014) vont dans le sens d'une territorialisation au profit des éleveurs dans leur ensemble. L'approche de Lefebvre en termes de production de l'espace est mise à profit ici pour montrer que les situations ne sont jamais figées. Les marges de manœuvres spatiales et sociales sont importantes pour repenser l'organisation harmonieuse des différents régimes d'activité. Acteurs locaux, décideurs politiques et ONG ont en l'occurrence la possibilité de s'engager plus avant dans les rapports de force pour influer sur les processus de territorialisation. II est possible de préserver une certaine fluidité à l'intérieur d'espaces pastoraux délimités et contrôlés par des instances locales reconnues. Les mobilités à la fois locales et régionales seront ainsi sécurisées et la résilience des systèmes d'élevage ouest-africain renforcée.

\section{Bibliographie}

Aldige, E. (1919), Situation de l'élevage et disponibilité en viande de l'Afrique Occidentale Française, Paris, Larose, $131 \mathrm{p}$.

D’Aquino, P . (2000), "L'agropastoralisme au nord du Burkina Faso (province du Soum) : une évolution remarquable mais encore inachevée», Autrepart, $\mathrm{n}{ }^{\circ} 15-16$, p. 29-47.

Audouin, S. et Gonin, A. (2014), «L'anacarde : produit de la globalisation, moteur de la territorialisation. L'exemple du sud du Bur kina Faso", EchoGéo, [EN LIGNE].

Augusseau, X., Cheylan, J.-P., et Liehoun, E. (2004), «Dynamiques territoriales de l'agropastoralisme en zone de migration: niveaux d'organisation et interactions", Cahiers Agricultures, n 6, p. 488-94.

Barrière, O. et Rochegude, A., éd. (2008), Foncier et environnement en Afrique. Des acteurs au(x) $\operatorname{droit}(s)$, Paris, Karthala, $427 \mathrm{p}$.

Bassett, T.J. et Turner, M.D. (2007), «Sudden shift or migratory drift? Fulbe herd movements to the Sudano-Guinean region of West Africa», Human Ecology, n' ${ }^{\circ}$ 5, p. 33-49.

Behnke, R.H., Scoones, I., et Kerven, C. (1993), Range ecology at disequilibrium: new models of natural variability and pastoral adaptation in African savannas, London, Overseas Development Ins titute, $248 \mathrm{p}$. 
Benoit, M. (1982), Nature peul du Yatenga, remarques sur le pastoralisme en pays Mossi, Paris, Orstom éditions, $82 \mathrm{p}$.

Bonfiglioli, A.M. (1990), «Pastoralisme, agro-pastoralisme et retour, itinéraires sahéliens», Cahiers des sciences humaines, $n^{\circ} 1-2$, p. 255-66.

Bonnet, B. (2014), "Vulnérabilité pastorale et politiques publiques de sécurisation d e la mobilité pastorale au Sahel», Mondes en dé veloppement, n 4, p. 7 1-91.

Boureima, A. et Boutrais, J. (2012), "Logiques pastor ales et de conservation de la nature : les transhumances et le Parc du W (Niger, Burkina Faso, Bénin)", Autrepart, n 60, p. 55-75.

Boutrais, J. (1994), "Pour une nouvelle cartographie des Peuls», Cahiers d'études africaines, $\mathrm{n}^{\circ}$ 133/135, p. $137-46$.

Breusers, M., N ederlof, S., et Van Rheenen, T. (1998), "Conflict or symbiosis? Disentangling farmerherdsman relations: the Mossi and Fulbe of the Central Plateau, Burkina Faso", The Journal of Modern African Studies, $\mathrm{n}^{\circ}$ 3, p. 357-8 0 .

Brottem, L., Turner, M.D., Butt, B., et al. (2014), «Biophysical Variability and Pastoral Rights to Resources:West African Transhumance Revisited», Human Ecology, n 3, p. 351-65.

Chauveau, J.-P., Le Pape M., et Olivier de Sardan, J.-P. (2 001), «La pluralité des normes et leurs dynamiques en Afrique» In Winter G., éd. Inégalités et politiques publiques en Afrique. Pluralité de normes et jeux d'acteur, Paris, IRD éditions, p. 145-62.

Colin de Verdière, P. (1995), Étude comparée de trois systèmes agropastoraux dans la région de Filingué (Niger); les conséquences de la sédentarisation de l'élevage pastoral au Sahel, Thèse de doct orat, Paris, INA, $220 \mathrm{p}$.

Doutressoulle, G. (1947), L'élevage en Afrique occidentale française, Paris, Larose, 298 p.

Dugué, P., Vall, E., Lecomte, P., et al. (2004), «Évolution des relations entre l'agriculture et l'élevage dans les savanes d'Afrique de l'Ouest et du Centre», Oléagineux, corps gras, lipides, n ${ }^{\circ} 4, \mathrm{p}$. 268-76.

Fanchette, S. (2011), Au pays des Peuls de Haute-Casamance: l'intégration territoriale en question, Paris, Karthala, $395 \mathrm{p}$.

Foucault, M. (1976), Histoire de la sexualité. Tome 1: La volonté de savoir, Paris, Gallimard, 211 p.

Gallais, J. (1972), «Les sociétés pastorales ouest-africaines fac e au développement», Cahiers d'études africaines, $n^{\circ} 47$, p. 353-68.

Gallais, J. (1976), Stratégies pastorales et agricoles des sahéliens dura nt la sécheresse 1969-1974, $P$ aris, Travaux et documents du CEGET, CNRS.

Gallais, J. (1984), Hommes du Sahel. Espaces-Temps et Pouvoirs. Le Delta inté rieur du Niger, Paris, Flammarion, $289 \mathrm{p}$.

Gautier, D., Ankogui-Mpoko, G.- F., Réounodji, F., et al. (2005), «Agriculteurs et éleveurs des savanes d'Afrique centrale: de la coexistence à l' intégration territoriale», L' Espace géographique, $n^{\circ} 3, p$. 223-36.

Gautier, D., Bonnerat, A., et Njoya, A. (2005), «The relationship between herders an d trees in space and time in northern Cameroon", Geographical Journal, n 4, p. 324-39.

Gonin, A. (2014), Jeux de pouvoir pour l'accès aux ressources et devenir de l'élevage en Afrique soudanienne : le foncier pastoral dans l'Ouest du Burkina Faso, Thèse de doctorat, Paris, université Paris 1 Panthéon-Sorbonne, $484 \mathrm{p}$.

Gonin, A. et Tallet, B. (2012)(a), "Quel avenir pour l'élevage dans le bassin cotonnier de l'Ouest du Burkina Faso ? Dynamiques agro-pastorales et recomposition s territoriales", Autrepart, $n^{\circ} 60, p$. 95-110.

Gonin, A. et Tallet, B. (2012)(b), "Changements spatiaux et pratiques pastorales : les nouvelles voies de la transhumance dans I 'Ouest du Burkina Faso", Cahiers Agricultures, n 6, p. 448- 54. 
Hagberg, S. (200 1), «À l'ombre du conflit violent», Cahiers d'études africaines, $n{ }^{\circ}$ 161, p . 45-72.

Hardin, G. (1968), «The Tragedy of the Commons», Science, n 3859, p. 1243- 8.

Inter-réseaux, (2015), «Le paradoxe de l'élevage au Sahel : forts enjeux, faibles soutiens», [E $n$ ligne].

Jullien, F. (2006), «Nomadisme et transhumance, chronique d'une mort annoncée ou voi e d'un développement porteur?», Afrique contemporaine, n² 217, p. 55-75.

Kintz, D. (1982), «Pastoralisme, agro-pastoralisme et organisation foncière: le cas des Peuls», I n: Le Bris E., Le Roy É., et Leimdorfer F ., éd. Enjeux fonciers en Afrique Noire, Paris, Orstom éditions, p. 2 12-7.

Kitchell, E., Turner M.D., et McPeak J.G. (2014), «Mapping of pastor al corridors: practices and politics in e astern Senegal», Pastoralism: Research, Policy and Practice, $\mathrm{n}^{\circ}$ 4, p. 17.

Kohler, J.-M. (1972), Les migrations des Mossi de l'Ouest, Paris, Orstom éditions, 106 p.

Lavigne-Delville, P. et Chauveau, J.-P., éd. (1998), Quelles politiques foncières pour l'Afrique rura le ? : réconcilier $p$ ratiques, légitimi té et légalité, Pa ris, Karthala, $745 \mathrm{p}$.

Lefebvre, H. (1974), La production de l'espace, Paris, Anthropos, 485 p.

Marty, A., Eberschweiler, A., et Dangbet, Z. (2009), Au cœur de la transhumance: Un campement chamelie $r$ au Tchad central, Paris, Orléans, Karthala, Iram, Antea, 277 p.

Le Meur, P.-Y. et Hochet, P. (2010), «Property Relations by other Means: Conflict over Dryland Resources in Ben in and Mali», European Journal of Development Research, n` 5, p. 643-59.

Milligan, S. et Binns, T. (2007), «Crisis in policy, policy in cris is: understanding environmental discourse and resource-use conflict in northern Nigeria», The Geographical Journal, n² 2, p. 143-56.

Monod, T. (1975), «Introduction au XIII ${ }^{\mathrm{e}}$ séminaire de I'International Afric an Institute», In: Pastoralism in Tropical Africa. Oxford, Oxford University Press, p. 3-98.

Moritz, M. (2006), "Changing contexts and dynamics of farmer-herder conflict s acr oss Wes t Africa», Canadian Journal of African Studies/La Revue canadienne des études africaines, $n^{\circ}$ 1, p. 1-40.

Moritz M., Catherin e B.L., Drent A.K., et al. (2013), «Rangeland governance in a open system: Protecting transhumance corridors in the Far North Province of Cameroon», Pastoralism: Research, Policy and Practice, $\mathrm{n}^{\circ} 26$, [En ligne].

Moutari, E. M. et Giraut, F. (2013), "Le corridor de transhumance au Sahel: un archétype de territoire multisitué?», L'Espace géographique, $\mathrm{n}^{\circ}$ 4, p. 306-23.

Niamir-Fuller, M. (1998), “The resilience of pastoral herding in Sahelian Africa», In Bekes F. et Folke C., éd. Linking social and ecological systems : Management practices and social mechanisms for building resilience. Cambridge, Cambridge University Press, p. 250-84.

Niamir-Fuller, M., éd. (1999), Managing Mobility in African Rangelands. London, Intermediate Technology Publications, $314 \mathrm{p}$.

Peluso, N.L. (2005), «Seeing property in land use : Loc al territorializati ons in West Kali mantan, Indonesia", Geografisk Tidsskrift-Danish Journal of Geography, n 1, p. 1-1 5.

Raffestin, C. (1980), Pour une géographie du pouvoir, Paris, Litec, 249 p.

Retaillé, D. (1989), «Mobilités des populations sahéliennes pendant la sécheresse aggravée de 1984 », In: Bret B., éd. Les hommes face aux sécheresses, Paris, IHEAL, p. 277-85.

Retaillé, D . (20 05), «L'espace mobile», In Antheaume B. et Giraut F., éd. Le territoire est mort. Vive les territ oires !, Paris, IRD éditions, p. 175-202.

Retaillé, D. (2011), «Du paradigme sahélien du lieu à l'espace (mondial) mobile», L'Information géographique, $\mathrm{n}^{\circ}$ 1, p. 71-85.

Retaillé, D. et Walther O. (2013), «L'actualité sahélo-saharienne au Mali : une invitation à penser l'espace mobile", Annales de géographie, n 694, p. 595-618. 
Richard, Y. (2007), «Les évolutions de l'espace en Russie», In Ciattoni A., éd. La Russie, Paris, Sedes, p. 111-42.

Sack, R.D. (1983), "Human territoriality: a theory», Annals of the Association of American Geographer $s, n^{\circ} 1$, p. 55-74.

Sack, R.D. (1986), Human territoriality: its theory and history, Cambridge, Cambridge University Press, $259 \mathrm{p}$.

Savonnet, G. (1968), Atlas de Haute-Volta, Ouagadougou, CVRS.

Schlager E. et Ostrom, E. (1992), «Property-rights regimes and natural resources: a conceptual analysis», Landeconomics, $\mathrm{n}^{\circ}$ 3, p. 24 9-62.

Scoones, I. (1994), Living with uncertainty: new directions in pastoral development in Africa, London, Intermediate Technology Publications, $210 \mathrm{p}$.

Thébaud, B. (2001), «Droit de communage ("commons ") et pastoralisme au Sahel : quel avenir p our I es éleveurs sahéliens ?», In Benjaminsen T.A. et Lund C., éd. Politics, Property and Production in the West African Sahel: Understanding Natural Resources Management, Stockholm, Nordic Africa Institute, p. 163-81.

Thébaud, B. (2002), Foncier pastoral et gestion de l'espace au Sahel: Peuls du Niger oriental et du Yagha burkinabé, Paris, Karthala, $343 \mathrm{p}$.

Thibaud, B. (2005), «Le pays dogon au Mali : de l'enclavement à l'ouverture?», Espace populations sociétés, $\mathrm{n}^{\circ} 1$, p. 4 5-56.

Turner, M.D. et Hiernaux, P. (2008), "Changing access to labor, pastures, and knowledge: The extensification of grazing management in Sudano-Sahelian West A frica ", Human Eco logy, $\mathrm{n}^{\circ}$, p. $59-80$.

Vall, É., Dugué, P., et Blanchard M. (2006), «Le tissage des rela tions agriculture-élevage au fil du coton», Cahiers Agricultures, ${ }^{\circ}$ 1, p. 72-9.

Vall, E., Koutou, M., Blanchard, M., et al. (2012), «Intégration agriculture-élevage et intensification écologique dans les systèmes agrosylvopast oraux de l'Ouest du Burkina Faso, province du Tuy", In Vall É., Andrieu N., Chia E., et al., éd. Partenariat, modélisation, expérimentations: quelles leçons pour la conception de l'innovation et l'intensification écologique?, Bobo-Dioulasso, ASAP, [cdrom].

Vandergeest, P. et Peluso, N.L. (1995), «Territorialization and state power in Thailand», Theory and society, ${ }^{\circ} 3$, p. 385-426.

Welte, T. (1997), «La grande transhumance», In Bierschenk T. et Le Meur P.-Y., éd. Trajectoires peules au Bénin, Paris, Karthala, p. 77-108. 